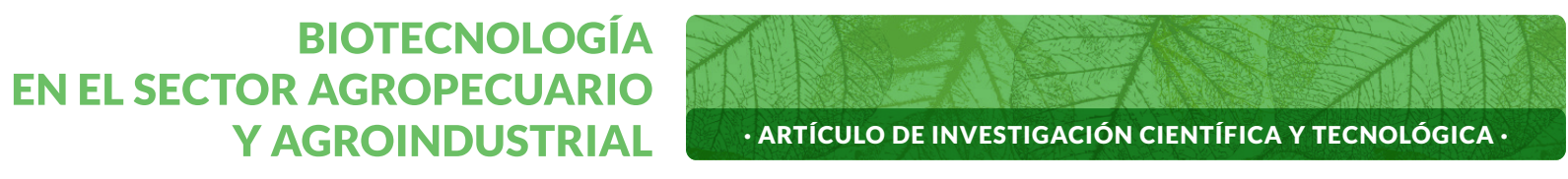

Vol. 19 No 1 · Enero - Junio 2021 · ISSN - 1692-3561 · ISSN-e 1909-9959 · DOI: https://doi.org/10.18684/BSAA(19)244-255

\title{
Evaluación agronómica de genotipos de Theobroma cacao L. en la Amazonia colombiana*
}

\section{Agronomic evaluation of Theobroma cacao L. genotypes in the colombian Amazon}

\section{Avaliação agronômica de genótipos de Theobroma cacao L. na Amazônia colômbiana}

\author{
GAMBOA-TABARES, JEAN-ALEXÁNDER'; RODRÍGUEZ-ORTIZ, JAIME-ANDRÉS'; \\ GAMBOA-TABARES, ARMANDO ${ }^{3}$; DURÁN-BAUTISTA, ERVIN-HUMPREY4;
}

ROJAS-VARGAS, SORAIDA ${ }^{5}$

Historial del Artículo

Recibido para evaluación: 13 de Febrero 2020.

Aprobado para publicación: 23 de Octubre 2020.

* Título del proyecto de origen: “Desempeño agronómico, evaluación fitosanitaria y caracterización molecular de clones promisorios de Theobroma cacao L. provenientes de materiales vegetales élite presentes en sistemas productivos del Bajo Caguán, municipio de Cartagena del Chairá". Financiación: Departamento Administrativo de Ciencia, Tecnología e Innovación COLCIENCIAS, Universidad de la Amazonia y Comité de Cacaoteros de Remolinos del Caguán y Suncillas CHOCAGUÁN. Culminación: 2020.

1 Universidad de la Amazonia, Facultad de Ingeniería, Programa de Ingeniería Agroecológica, Grupo de Investigación en Entomología Universidad de la Amazonia (GIEUA). M.Sc. en Agricultura Ecológica. Florencia, Colombia. https://orcid.org/0000-0002-8783-0175

2 Universidad de la Amazonia, Facultad de Ciencias Básicas, Maestría en Ciencias Biológicas, Grupo de Investigación en Agroecosistemas y Conservación en Bosques Amazónicos (GAIA). Mg. en Ciencias Biológicas. Florencia, Colombia. https://orcid.org/0000-0003-0973-8549

3 Universidad de la Amazonia, Facultad de Ciencias Básicas, Maestría en Ciencias Biológicas. Mg. en Ciencias Biológicas. Florencia, Colombia. https://orcid.org/0000-0001-5777-8495

4 Universidad de la Amazonia, Facultad de Ingeniería, Programa de Ingeniería Agroecológica, Grupo de Investigación en Agroecosistemas y Conservación en Bosques Amazónicos (GAIA). Mg. en Agroforestería. Florencia, Colombia. https://orcid.org/0000-0002-4155-6350

5 Universidad de la Amazonia, Facultad de Ingeniería, Programa de Ingeniería Agroecológica, Grupo de Investigación en Agroecosistemas y Conservación en Bosques Amazónicos - GAIA Ingeniero Agroecólogo. Florencia, Colombia. https://orcid.org/0000-0002-4860-2954 


\section{RESUMEN}

Los clones universales de cacao (Theobroma cacao L.) introducidos en la región amazónica de Colombia, presentan bajos rendimientos. El objetivo de este trabajo fue evaluar el comportamiento agronómico de genotipos locales promisorios de T. cacao bajo las condiciones de la Amazonia Colombiana. El vivero y parcela experimental se establecieron en la vereda Cuba, municipio de Cartagena del Chairá. En la parcela experimental se implementó un diseño experimental Factorial 7 x 4, con Factor A (genotipos) y Factor B (distancias de siembra). En vivero se evaluó la altura y el diámetro del tallo del clon portainjerto IMC-67, y en la parcela experimental el prendimiento y desempeño agronómico de siete genotipos. El clon IMC-67 tuvo germinación del 95\%, velocidad de crecimiento de $0,200 \mathrm{~cm} \cdot$ día $^{-1}$, e incremento en diámetro del tallo de 0,003 cm.día ${ }^{-1}$. Los siete genotipos presentaron prendimiento del injerto del $95,73 \%$, sin diferencias estadísticamente significativas ( $p<0,05)$. La altura total $(\mathrm{cm})$, longitud de injerto $(\mathrm{cm})$, diámetro del portainjerto $(\mathrm{cm})$, número de hojas y área de copa $\left(\mathrm{m}^{2}\right)$, presentaron diferencias estadísticamente significativas ( $p<0,0001$ ). El genotipo RMC-4, presentó mayores valores de biomasa seguido de los genotipos RMC-2 e ICS-1, sin diferencias estadísticamente significativas $(p<0,05)$, entre los genotipos evaluados. Se concluye que los genotipos promisorios RMC-3, RMC-4 y RMC-5, presentan potencial para su consolidación como clones universales en el futuro.

\section{ABSTRACT}

The universal clones of cocoa (Theobroma cacao L.) introduced in the Colombian Amazon region, present low yields. The objective of this work was to evaluate the agronomic behavior of promissory local genotypes of T. cacao under the conditions of the Colombian Amazon. The nursery and experimental plot were established on the rural division of Cuba in Cartagena del Chairá town. A $7 \times 4$ Factorial Experiment was implemented, with Factor A (genotypes) and Factor $B$ (planting distances). In the nursery, height and stem diameter of the IMC-67 rootstock clone were evaluated, and in the experimental plot the agronomic yield and performance of seven genotypes. IMC-67 clone had $95 \%$ of

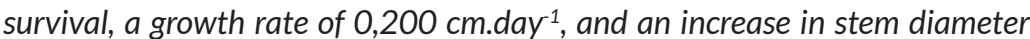
of 0,003 cm.day ${ }^{-1}$ The seven genotypes showed $95,73 \%$ of survival, without statistically significant differences $(p<0,05)$. The total height $(\mathrm{cm})$, graft length $(\mathrm{cm})$, rootstock diameter $(\mathrm{cm})$, number of leaves and treetop area $\left(\mathrm{m}^{2}\right)$, showed statistically significant differences ( $p<0,0001)$. RMC-4 genotype showed higher biomass values followed by the RMC-2 and ICS-1 genotypes, without sta-

Correspondencia: j.gamboa@udla.edu.co

Cómo citar este artículo: GAMBOA-TABARES, JEAN-ALEXÁNDER; RODRÍGUEZ ORTIZ, JAIME-ANDRÉS; GAMBOA-TABARES, ARMANDO; DURÁN-BAUTISTA, ERVIN-HUMPREY; ROJAS-VARGAS, SORAIDA. Evaluación agronómica de genotipos de Theobroma cacao L. en la Amazonia colombiana. Biotecnología en el sector agropecuario y agroindustrial, v. 19, n. 1, 2021, p. 244-255. Doi: https://doi. org/10.18684/BSAA(19)244-255

\section{PALABRAS CLAVE:}

Cacao; Agroforestal; Desarrollo; Caracterización; Theobroma cacao L.

\section{KEYWORDS:}

Cocoa; Agroforestry; Development; Characterization; Theobroma cacao L.

\section{PALAVRAS-CHAVE:}

Cacau; Agroflorestas; Desemvolvimento, Caracterização; Theobroma cacao L. 
tistically significant differences ( $p<0,05)$, among the genotypes evaluated. We concluded that the RMC-3, RMC-4 and RMC-5, present potential for consolidation as universal clones in the future.

\section{RESUMO}

Os clones universais de Theobroma cacao L. introduzidos na região amazônica colômbiana apresentam baixos rendimentos. O objetivo deste estudo foi avaliar o comportamento agronômico de genótipos locais promissores de T. cacao em condições da Amazônia colômbiana. O viveiro e a chácara experimental foram estabelecidos na Vereda Cuba, município de Cartagena del Chairá. Na chácara experimental foi implementado um experimento fatorial $7 \times 4$, com o fator $A$ (genótipos) e o fator $B$ (distâncias de plantio). No viveiro foi avaliado a altura e diâmetro do caule do clone porta-enxerto IMC67, e na chácara experimental a germinação e desempenho agronômico de sete genótipos. O clone padrão IMC67 teve 95\% de germinação, taxa de crescimento de 0,200 cm.dia ${ }^{-1}$ e aumento no diâmetro do caule de 0,003 $\mathrm{cm} \cdot$ dia $^{-1}$ os sete genótipos apresentaram germinação do caule do 95,73\%, sem diferenças estatisticamente significativas $(p<0,05)$. A altura total $(\mathrm{cm})$, comprimento do enxerto $(\mathrm{cm})$, diâmetro do porta-enxerto $(\mathrm{cm})$, número de folhas e área da copa $\left(\mathrm{m}^{2}\right)$, apresentaram diferenças estatisticamente significantes ( $\left.p<0,0001\right)$. O genótipo RMC4, apresentou maiores valores de biomassa, seguido pelos genótipos RMC2 e ICS1, sem diferenças estatisticamente significantes ( $p<0,05)$, entre os genótipos avaliados. Conclui-se que os promissores genótipos RMC3, RMC4 e RMC5 apresentam potencial para sua consolidação como clones universais no futuro.

\section{INTRODUCCIÓN}

El cacao (Theobroma cacao L.-Malvaceae) es un árbol frutal originario de la selva tropical húmeda en la cuenca del Amazonas [1]. La investigación genómica indica que la mayor diversidad de T. cacao se encuentra en el Noroeste de América del Sur, señalando esta región como su centro de origen [2]. El cacao fue llevado a Mesoamérica, donde el uso y domesticación pudo haber comenzado hace aproximadamente 3600 años [3]. El cultivo se expandió luego a otras regiones, notablemente en el siglo XIX, por lo que en la actualidad es producido en 40 países ubicados en la región tropical de África, Asia y América [4].

Costa de Marfil, Ghana, Indonesia, Brasil y Nigeria son los principales productores de cacao en el mundo [5]. Los granos de cacao se exportan principalmente a Europa y América del Norte para su procesamiento [6]. En el mercado internacional del cacao se comercializan el grano seco, residuos (cáscaras, cortezas, cascarillas y desechos de cacao), pasta, torta, manteca, polvo y chocolate [7]. Los mayores consumidores de productos de cacao son Estados Unidos (20\%), Alemania (9\%), Francia (6\%), Reino Unido (6\%), Brasil (5\%), Rusia (5\%) y Japón (4\%) [8]. En los países en que se cultiva, es una fuente de ingresos para pequeños agricultores, que dependen principalmente del trabajo familiar [9]. En Colombia, la cadena productiva del cacao está conformada por aproximadamente 35.000 familias en diferentes regiones del país, que generan aproximadamente 100.000 empleos en actividades de productos intermedios y finales [10]. Durante el año 2018, Colombia tuvo un área cosechada de 190469 hectáreas (ha) y una producción de 101019 toneladas (t), lo que indica un rendimiento promedio nacional de 0,53 t.ha ${ }^{-1}$; la participación del departamento del Caquetá en la producción nacional fue de 1,55\%, [11]. Durante el año 2019, el país alcanzó una producción de 59665 t de grano seco [12].

La práctica cultural dominante del cultivo de cacao en la Amazonia, implica la tumba y quema de bosques o barbechos, para sembrar especies de sombrío temporal como son el plátano (Musa x paradisiaca L.-Musaceae) y banano (Musa $x$ sapientum L.-Musaceae), y el establecimiento de maderables para sombrío definitivo. Con respecto a los materiales genéticos de cacao, las plantaciones de más de 30 años se establecieron con árboles de origen híbrido resultado de la polinización cruzada entre clones universales introducidos y árboles silvestres. Como innovación tecnológica en el sector, las nuevas plantaciones se han establecido principalmente con los clones TSH-565, ICS1, ICS-39, ICS-60, ICS-95, EET-8 y CCN-51, injertados en portainjertos de origen híbrido del clon IMC-67. 
En la región Amazónica se desconoce el recurso genético de T. cacao correspondiente a materiales autóctonos, adaptados a las condiciones edáficas y climáticas de la región. Personal técnico y científico del Comité de Cacaoteros de Remolinos del Caguán y Suncillas CHOCAGUÁN, seleccionaron a través del modelo de mejoramiento genético participativo, materiales promisorios de cacao provenientes de árboles híbridos de polinización abierta con buena adaptabilidad a los suelos, tolerancia a plagas y enfermedades y altos rendimientos en producción de grano seco, a los cuales se les asignaron los códigos RMC-1, RMC-2, RMC-3, RMC-4 y RMC-5. El objetivo de la presente investigación fue evaluar el comportamiento agronómico de cinco genotipos promisorios de Theobroma cacao L. establecidos en arreglo agroforestal, bajo las condiciones agroecológicas de la Amazonia colombiana.

\section{MÉTODO}

El vivero y la parcela experimental se establecieron en la vereda Cuba del corregimiento de Remolinos del Caguán, municipio de Cartagena del Chairá, departamento de Caquetá, ubicado a 00³1'53" de latitud norte y $74^{\circ} 17^{\prime} 48^{\prime \prime}$ de longitud oeste (Figura 1). El sitio presenta temperatura media de $29,5^{\circ} \mathrm{C}$, precipitación anual de $3800 \mathrm{~mm}$, humedad relativa del $84 \%$, brillo solar de 1700 horas. año-1, $183 \mathrm{~m}$ de altitud y corresponde a la zona de vida Bosque Húmedo Tropical [13].

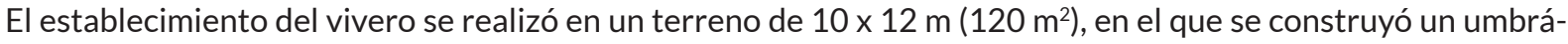
culo con polisombra al 75\%. Se prepararon $10 \mathrm{t}$ de sustrato para el llenado de las bolsas y posterior siembra de la semilla, cuyas proporciones fueron $94 \%$ de suelo (rico en materia orgánica obtenido en el predio), 3\% de mantillo de bosque o capote (fuente de microorganismos benéficos y materia orgánica), $2 \%$ de roca fosfórica (Fosforita Huila $\mathrm{P}_{2} \mathrm{O}_{5} 28 \%$ y CaO $40 \%$,) y $1 \%$ de cascarilla de arroz. Se usaron bolsas con capacidad de $2 \mathrm{~kg}$, con dimensiones de 0,15 $\mathrm{mm}$ de espesor, $10 \mathrm{~cm}$ de ancho y $30 \mathrm{~cm}$ de largo, con perforaciones y fuelle.

Figura 1. Ubicación geográfica del área de estudio.

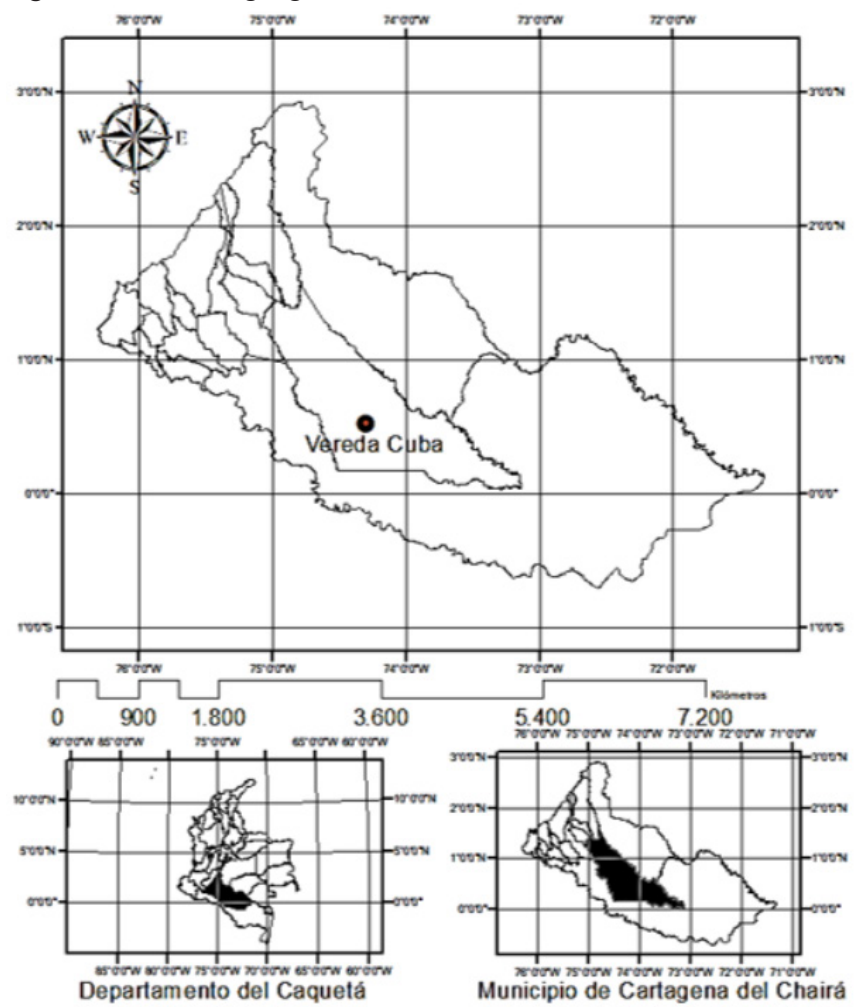


El material vegetal usado como portainjerto se obtuvo de semillas certificadas del clon IMC-67 suministradas por la Federación Nacional de Cacaoteros (FEDECACAO), procedentes del Municipio de San Vicente de Chucurí en el Departamento de Santander, las que estaban pregerminadas al momento de siembra. Se realizaron 6 muestreos de la altura $(\mathrm{cm})$ y diámetro a $1 \mathrm{~cm}$ del suelo $(\mathrm{cm})$ a cada planta, desde los 17 dds (días después de siembra) hasta los 123 dds, con intervalos de 20 días entre muestreos.

Finalizada la etapa de vivero, se seleccionaron las 2506 plantas que presentaron mayor altura y diámetro del tallo; éstas se llevaron a un campo experimental de 3 ha bajo condiciones de arreglo agroforestal, asociadas con plátano (Musa x paradisiaca) y abarco (Cariniana pyriformis Miers-Lecythidaceae). Se utilizó un Experimento Factorial 7x 4, en Diseño Completamente al Azar, con Factor A correspondiente a siete clones (RMC-1, RMC-2, RMC-3, RMC-4, RMC-5, ICS-1 e ICS-60) y Factor B que corresponde a cuatro distancias de siembra (3,0 x 3,0 m, 3,5 x 3,5 m, 3,0 x $4,0 \mathrm{~m}$ y 4,0 $\times 4,0 \mathrm{~m}$ ), para un total de 28 unidades experimentales. Cada unidad experimental correspondió a un área de $1056 \mathrm{~m}^{2}(24 \times 44 \mathrm{~m})$. Diez meses posteriores al trasplante del patronaje de cacao a sitio definitivo, las plantas se injertaron con los genotipos promisorios (RMC-1, RMC-2, RMC-3, RMC-4, RMC-5) y clones universales (ICS1 e ICS-60) mediante técnica de injerto lateral o aproximación. El material vegetal fue obtenido del jardín clonal a pequeña escala (JCPE) de propiedad del Comité de Cacaoteros de Remolinos del Caguán y Suncillas (CHOCAGUÁN), ubicado en el corregimiento de Remolinos del Caguán, previa estimulación de los árboles mediante poda y fertilización. Transcurridos 30 días desde la injertación, se evaluó el resultado del prendimiento de los injertos para cada uno de los genotipos de cacao, el cual consistió en determinar el estado del material injertado de cada planta, clasificado en yemas latentes (tejido vivo sin desarrollo foliar), brote (inicio de desarrollo de la yema) o muerta (yemas muertas), y se determinó el porcentaje de prendimiento total para cada subparcela experimental.

Para la evaluación agronómica de los clones (promisorios y universales), se desarrollaron dos muestreos no destructivos mediante la selección aleatorizada de cinco plantas por unidad experimental. El primer muestreo se realizó 20 meses posteriores al trasplante en campo (13 meses después de la injertación) y el segundo muestreo 34 meses posteriores a trasplante ( 27 meses después de la injertación). También, se realizó muestreo destructivo a los 27 meses posteriores a injertación, a una planta por unidad experimental. La biomasa total de las plantas se pesó en fresco (peso húmedo) con balanza de $0,5 \mathrm{~kg}$ de precisión, se tomó una muestra de aproximadamente $250 \mathrm{~g}$ de cada una de las partes de la planta (tallo, raíz y hojas) y se secó en horno a $60^{\circ} \mathrm{C}$ durante 48 horas, posteriormente se estimó el peso seco total de cada parte a partir del porcentaje de humedad calculado. Las variables evaluadas para desempeño agronómico fueron: altura $(\mathrm{cm})$, longitud de injerto $(\mathrm{cm})$, diámetro de portainjerto $(\mathrm{cm})$, número de hojas, área de copa $\left(\mathrm{m}^{2}\right)$ y peso seco $(\mathrm{g})$.

Las variables agronómicas estudiadas (altura y diámetro del tallo a $1 \mathrm{~cm}$ del suelo) en la fase de vivero, se estimaron a partir de la pendiente de regresión lineal respecto al tiempo de evaluación (17, 41, 62, 79, 104 y 123 días después de siembra), y se determinó la relación entre las variables, mediante coeficientes de correlación de Pearson. A las variables de prendimiento de injertos (\% de yemas en estado latente, brote y muerto) se les verificaron los supuestos de normalidad y homocedasticidad con las pruebas de Shapiro-Wilks y Levene, respectivamente, y se analizaron mediante prueba de análisis de varianza (ANDEVA) y se realizó una prueba de comparación de medias, en función de los genotipos evaluados, mediante la prueba de comparaciones múltiples LSD de Fisher $(p=0,05)$. Todas las pruebas estadísticas se realizaron en el programa InfoStat versión 2018 [14].

Con las variables altura total $(\mathrm{cm})$, longitud de injerto $(\mathrm{cm})$, diámetro del portainjerto $(\mathrm{cm})$, número de hojas y área de copa $\left(\mathrm{m}^{2}\right)$ se realizó un análisis de conglomerados por el método de Ward y la distancia Euclídea; además, se ajustó un modelo lineal general mixto (MLGM) $\left[Y_{i \mathrm{ij}}=\mu+A_{i}+B_{i}+(A B)_{i j}+e_{i j k}\right]$ para analizar el efecto de los factores fijos: genotipo (A: siete genotipos) y distancia de siembra (B: cuatro distancias) con medidas en el tiempo (dos muestreos: 20 y 34 meses después de siembra), y el efecto de sus interacciones ( $A B$ ). El efecto de las parcelas y subparcelas asociadas a las distancias de siembra dentro de los genotipos se incluyeron como efectos aleatorios. Para las variables longitud de injerto y diámetro de copa, la varianza residual se modeló para contemplar varianzas diferentes (Heterocedasticidad, H) por muestreo y genotipo respectivamente, mientras que la correlación residual para las observaciones sucesivas realizadas sobre una misma planta (ubicada dentro de una subparcela 
y ésta en una parcela) se examinó mediante un modelo autorregresivo de orden 1 (AR1). Los criterios de Akaike (AIC), Bayesiano (BIC) y LogLik se utilizaron para la selección de la estructura de varianzas y correlaciones residuales [15]. El análisis se realizó utilizando la función Ime en el paquete nIme [16] en el software R, versión 3.4.4 [17] y utilizando la interfaz en el software Infostat v. 2018p [14]. Las diferencias entre las medias de las variables en todos los factores fijos se analizaron con la prueba LSD de Fisher con una significación de $p=0,05$.

\section{RESULTADOS}

\section{Crecimiento del clon IMC-67}

La germinación del clon IMC-67 fue del 95\%, lo cual se atribuye a varios factores entre los que se resalta la composición del sustrato, utilización de semillas pregerminadas y las condiciones óptimas del vivero. El índice de desarrollo promedio fue de $0,200 \mathrm{~cm}$.dia ${ }^{-1}$ durante los 123 días después de siembra (dds), con menor valor entre los 62 y $79 \mathrm{dds}$, con un incremento de solo $0,097 \mathrm{~cm} \cdot \mathrm{dia}^{-1}$ y mayor entre 0 y $17 \mathrm{dds}$ con un incremento de $0,680 \mathrm{~cm}^{-d_{i a}{ }^{-1}}$ (Figura 2A). El diámetro a $1 \mathrm{~cm}$ de altura, tuvo un incremento de $0,003 \mathrm{~cm}^{-1 i a^{-1}}$, con mayor valor de 104 a 123 dds con 0,096 cm.dia ${ }^{-1}$ y menor valor de 79 a 104 dds con 0,037 cm.dia-1 (Figura 2B). En el clon IMC-67 se tuvo un incremento de $0,240 \mathrm{~cm}$.día ${ }^{-1}$ para la altura y de $0,002 \mathrm{~cm} \cdot$ día $^{-1}$ para la circunferencia del tallo [18]; otros autores reportaron a los $90 \mathrm{dds}$ un diámetro superior $(0,710 \mathrm{~cm})$ al encontrado en este estudio $(0,580 \mathrm{~cm})$ a los $104 \mathrm{dds}$, debido al uso de rizobacterias [19]. En la figura $2 \mathrm{C}$ se presenta la correlación entre las variables altura y diámetro del tallo para el clon IMC-67 en condiciones de vivero. El diámetro de la planta es importante en el desarrollo, ya que es el lugar de almacenamiento de nutrientes esenciales que darán lugar a plantas vigorosas y productivas [20].

Figura 2. Crecimiento del clon de portainjerto IMC-67 bajo condiciones de vivero. A. Altura. B. Diámetro. C. Regresión lineal Altura vs. Diámetro.
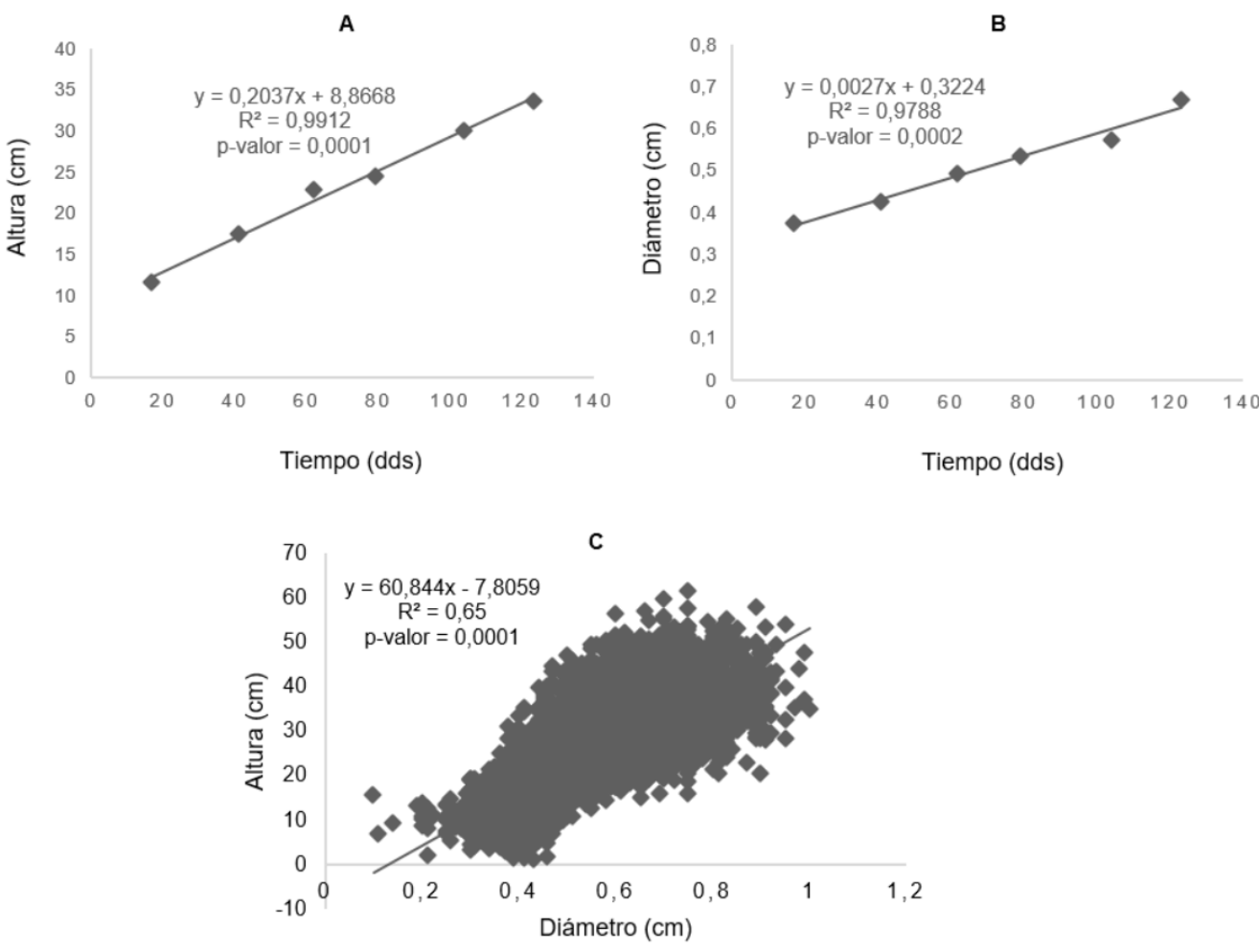


\section{Compatibilidad portainjerto-injerto}

Los genotipos promisorios regionales y clones universales testigos, presentaron un porcentaje de prendimiento del $95,73 \%$, sin diferencias estadísticamente significativas $(p<0,05)$ (Figura 3). Los resultados altos en el prendimiento de injertos están relacionados con las buenas condiciones del material vegetal empleado, y están influenciados por factores como la adecuada ejecución de los procedimientos, condiciones de la planta portainjerto, la adecuada alineación de tejidos parenquimatosos, las buenas prácticas agrícolas y la habilidad de los injertadores [21].

Figura 3. Compatibilidad de injertos para genotipos promisorios y clones universales injertados en el clon IMC-67. Letras diferentes indican diferencias estadísticamente significativas según LSD Fisher $(p \geq 0,05)$.

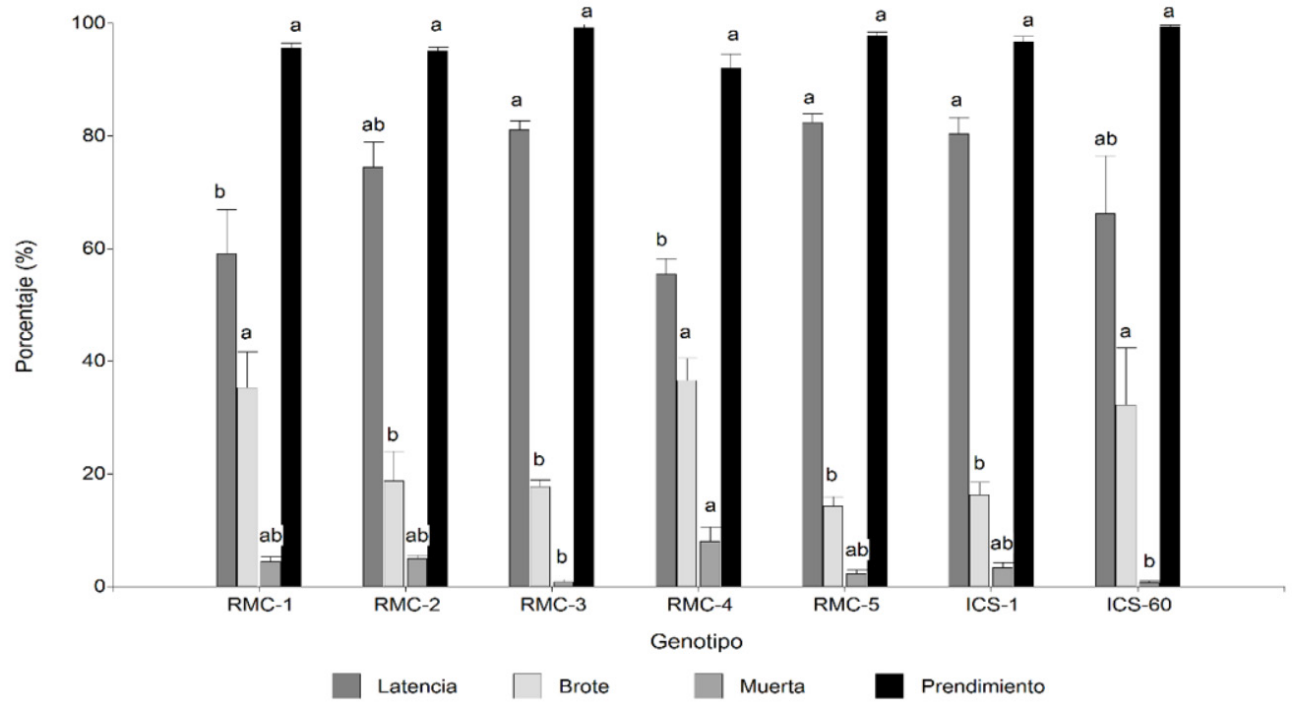

En un estudio anterior, determinaron que el porcentaje ideal de prendimiento obtenido con clones ICS-1, ICS39, ICS-60, ICS-78, ICS-95, TSH-565, TSH-812, IMC-67, CCN-51, EET-8, SCC-59 y SCC-61, injertados mediante la técnica en parche, debe estar alrededor del $95 \%$ en portainjertos mayores de 60 días [22], lo que coincide con el presente estudio, dónde la mayoría de los genotipos presentaron prendimiento superior al $95 \%$, lo que representa una buena compatibilidad entre el material injertado y el patronaje utilizado, sobresaliendo el clon universal de referencia ICS-60 (99,45\%) y los genotipos promisorios regionales RMC-3 y RMC-5 (99,17 y 97,51\%).

La variable de latencia osciló entre 55,62 y $82,83 \%$, siendo menor en el genotipo RMC-4 y mayor en el RMC5 , respectivamente, con diferencias estadísticamente significativas $(p<0,05)$. Con respecto a la mortalidad, se observó que el material RMC-4 mostró el mayor valor (7,12\%) seguido por el RMC-2 (5,01\%) sin diferencias estadísticamente significativas $(p<0,05)$, y los de menor valor fueron materiales ICS-60 y RMC-3 con 0,55 y 0,83\% respectivamente. Diversos estudios permiten deducir que las diferencias en la formación de brotes en relación con la técnica de injerto, son atribuidas a los factores nutricionales y la cantidad de carbohidratos en la plántula.

\section{Desempeño agronómico de genotipos}

Las variables agronómicas a los 20 y 34 meses después de la siembra (mds), obtenidas para los factores muestreo (M), genotipos (G), distancias de siembra (DS) y las interacciones (MxG, GxDS, MxDS y MxGxDS), evidenció que para los factores $M$ y G, las variables altura total $(\mathrm{cm})$, longitud de injerto $(\mathrm{cm})$, diámetro del portainjerto $(\mathrm{cm})$, número de hojas y área de copa $\left(\mathrm{m}^{2}\right)$, presentaron diferencias estadísticamente significativas $(p<0,0001)$. Por otra parte, se presentaron diferencias estadísticamente significativas entre distancias de siembra para la 
variable diámetro del portainjerto. En cuanto a las interacciones se presentaron diferencias estadísticamente significativas $(p<0,05)$ en $M \times G$ para todas las variables $(p<0,0001)$, en $G \times D S$ para las variables altura total, diámetro de portainjerto y número de hojas, en Mx DS para la variable longitud de injerto, y en $\mathrm{M} \times \mathrm{G} \times \mathrm{DS}$ para las variables diámetro de portainjerto y número de hojas (Cuadro 1).

Cuadro 1. Análisis de varianza de los efectos muestreo (M), genotipo (G), distancia de siembra (DS) y sus interacciones (MxG, GxDS, MxDS y MxGxDS), para variables agronómicas de genotipos a los 34 meses después de la siembra.

\begin{tabular}{|l|l|l|l|l|l|l|l|}
\hline \multirow{2}{*}{ Variables* } & \multicolumn{7}{|c|}{ p-valores basados en el estadístico de LSD Fisher } \\
\cline { 2 - 8 } & \multicolumn{1}{|c|}{ M } & \multicolumn{1}{|c|}{ G } & \multicolumn{1}{c|}{ DS } & \multicolumn{1}{c|}{ M x G } & \multicolumn{1}{c|}{ G x D } & \multicolumn{1}{c|}{ M x DS } & M x G x DS \\
\hline AT & $<0,0001$ & $<0,0001$ & 0,1564 & $<0,0001$ & 0,0250 & 0,2317 & 0,0757 \\
\hline LI & $<0,0001$ & $<0,0001$ & 0,1219 & $<0,0001$ & 0,3895 & 0,0434 & 0,0538 \\
\hline DP & $<0,0001$ & $<0,0001$ & 0,0015 & $<0,0001$ & 0,0002 & 0,3539 & 0,0017 \\
\hline NH & $<0,0001$ & $<0,0001$ & 0,6683 & $<0,0001$ & $<0,0001$ & 0,6613 & $<0,0001$ \\
\hline AC & $<0,0001$ & $<0,0001$ & 0,1507 & $<0,0001$ & 0,2168 & 0,2131 & 0,4306 \\
\hline
\end{tabular}

*Altura total (AT), longitud de injerto (LI), diámetro del portainjerto (DP), número de hojas (NH) y área de copa (AC).

Para el factor genotipo, se encontraron diferencias estadísticamente significativas $(p<0,05)$ en todas las variables para los dos momentos de evaluación, sobresaliendo en desempeño los genotipos RMC-3 y RMC-4. Los genotipos RMC-2 e ICS-60 presentaron los valores más bajos respecto a las variables evaluadas, resultando estadísticamente diferentes $(p<0,05)$. Con respecto al factor distancias de siembra, se presentaron diferencias estadísticamente significativas $(p<0,05)$ para las variables evaluadas entre genotipos y tiempos de muestreo, excepto para la variable número de hojas, que no presentó diferencias estadísticas significativas entre los genotipos. La distancia que tuvo mayor efecto positivo sobre las variables evaluadas fue D4 (4,0 x 4,0 m) (Cuadro 2).

El análisis de conglomerados permitió reconocer tres grupos bien diferenciados. El grupo 3 conformado por los genotipos RMC-2 (D4, D3, D2, D1), RMC-1 (D3), RMC-3 (D2), ICS-60 (D4, D3, D2, D1) y RMC-5 (D2) de bajo desempeño agronómico y con el menor número de hojas. El grupo 1 con desempeño agronómico medio y asociado a las variables diámetro de copa y diámetro de portainjerto conformado por los genotipos ICS-1 (D1, D2 y D3), RMC-4 (D1, D2, D3, D4), RMC-5 (D3, D4), RMC-3 (D1), RMC-1 (D2 y D4) y finalmente, el grupo 2 de alto rendimiento agronómico y con mayor longitud de injerto y altura total conformado por los genotipos RMC-5 (D1), RMC-1 (D1), RMC-3 (D4, D3) e ICS-1 (D4) (Figura 4).

El genotipo RMC-4, presentó los mayores valores de biomasa para las variables peso seco de hojas (PSH 610,5 g), peso seco tallo (PST 1890,6 g) y peso seco total (PSTa $2816 \mathrm{~g}$ ), seguido de los genotipos RMC-2 (579, 1125 y 2059 g, respectivamente) e ICS-1 (400,6, 1437 y 2231 g, respectivamente), sin diferencias estadísticamente significativas $(p<0,05)$. Los menores valores de biomasa se presentaron en los genotipos RMC-1 y RMC-3, seguido de ICS-60 (Figura 5). Estos resultados guardan una relación con los resultados de las variables agronómicas, en cuanto a que los materiales RMC-4 e ICS-1 presentaron en promedio los mayores valores.

Los genotipos RMC-4 e ICS-1 tienen mayor capacidad de fijar C en su biomasa respecto a los demás materiales, lo que representa un valor agregado para los productores de cacao que los adopten en sus sistemas productivos. Los compartimentos de los sistemas agroforestales (SAF) de T. cacao que más carbono almacenan son: suelo (52,61\%), biomasa aérea (plantas de cacao y árboles de sombra) (35,86\%), raíces gruesas (7,52\%), raíces finas $(4,15 \%)$, necromasa $(0,32 \%)$ y hojarasca $(0,32 \%)$ [23]. En un estudio anterior, determinaron para sistemas agroforestales de cacao, un almacenamiento de 154,91 y 344,24 t C.ha ${ }^{-1}$ a los 8 y 16 años de establecidos, respectivamente [24]. Es de mencionar que, la cantidad de $C$ almacenado por un SAF depende de la abundancia de árboles asociados, su estructura y área basal [25]. 
Cuadro 2. Desempeño agronómico de genotipos promisorios y clones universales en arreglo agroforestal durante fase de establecimiento.

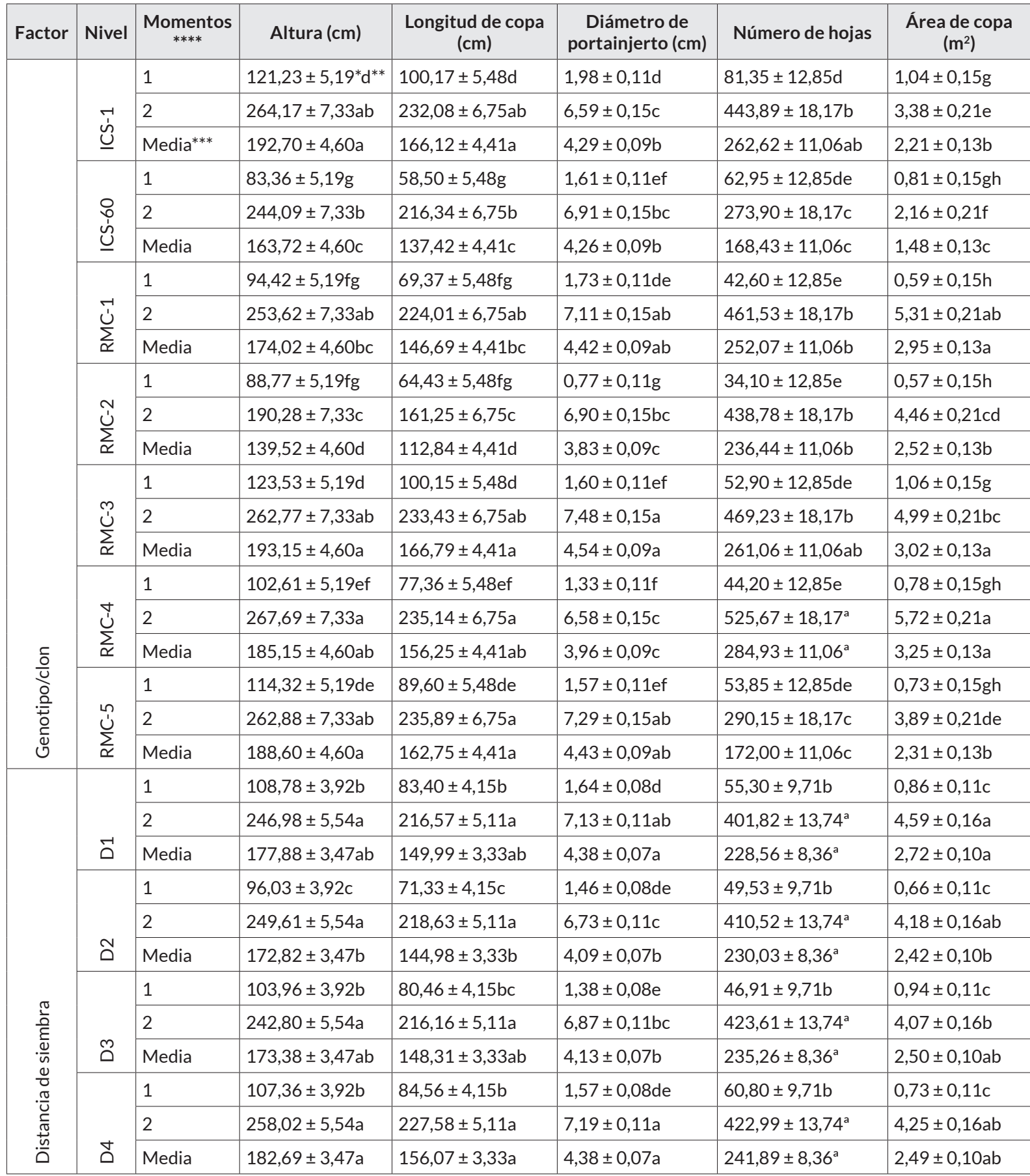

* Error estándar

** Valores en la columna seguidos por la misma letra no difieren estadísticamente entre tiempos para cada nivel del factor (medias separadas por la prueba LSD, $p \geq 0,05$ )

*** Medias en cada columna seguidas por la misma letra no difieren estadísticamente entre niveles del factor (Prueba LSD, $p \geq 0,05)$.

**** Momentos de muestreo: 1) 20 meses después de siembra, 2) 34 meses después de siembra. 
Figura 4. Análisis multivariado (conglomerados método Ward y distancia Euclídea) para variables agronómicas de genotipos a diferentes distancias de siembra.

\section{Ward}

Distancia: (Euclidea)

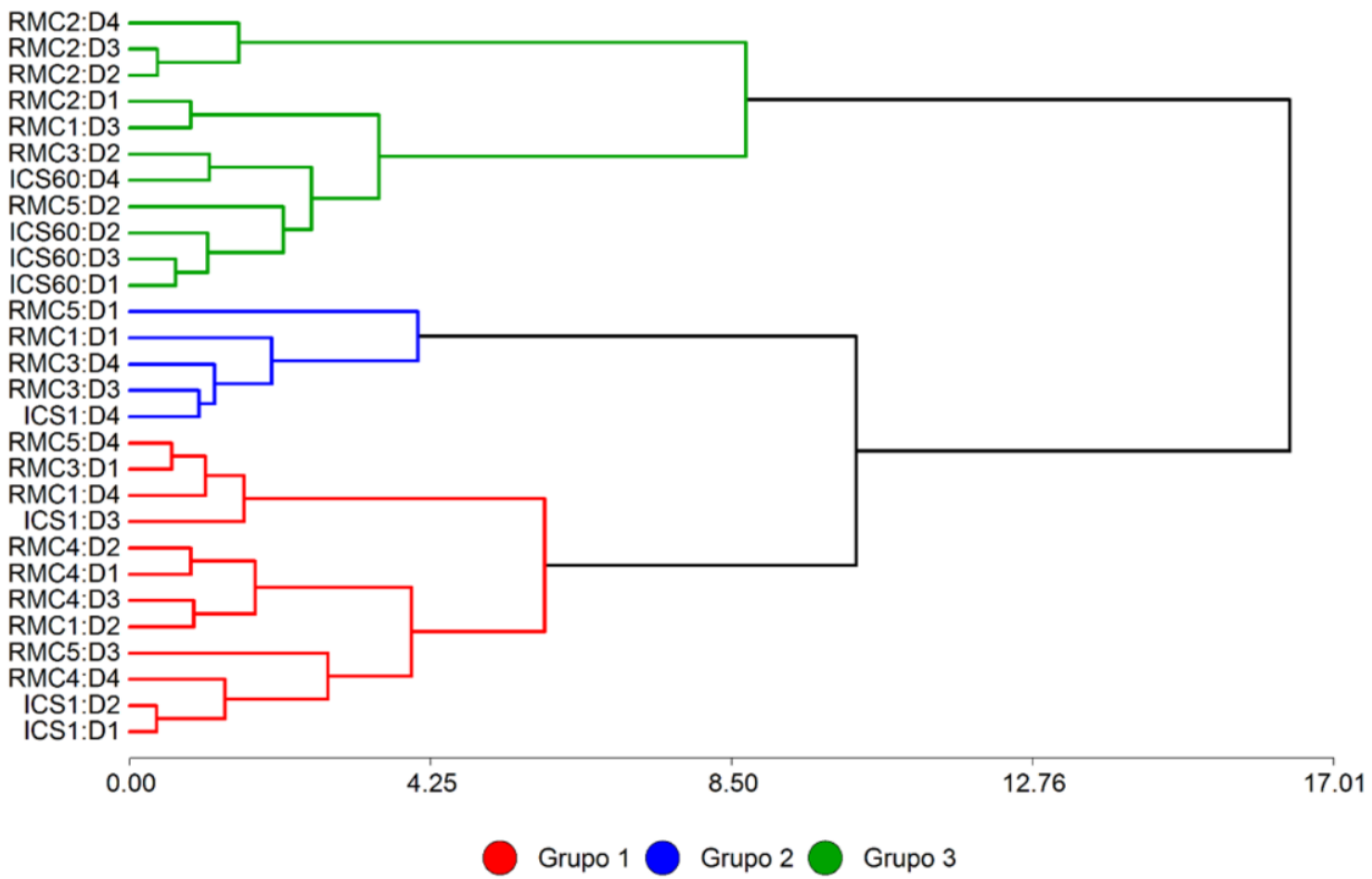

Figura 5. Producción de biomasa de genotipos establecidos en arreglo agroforestal.

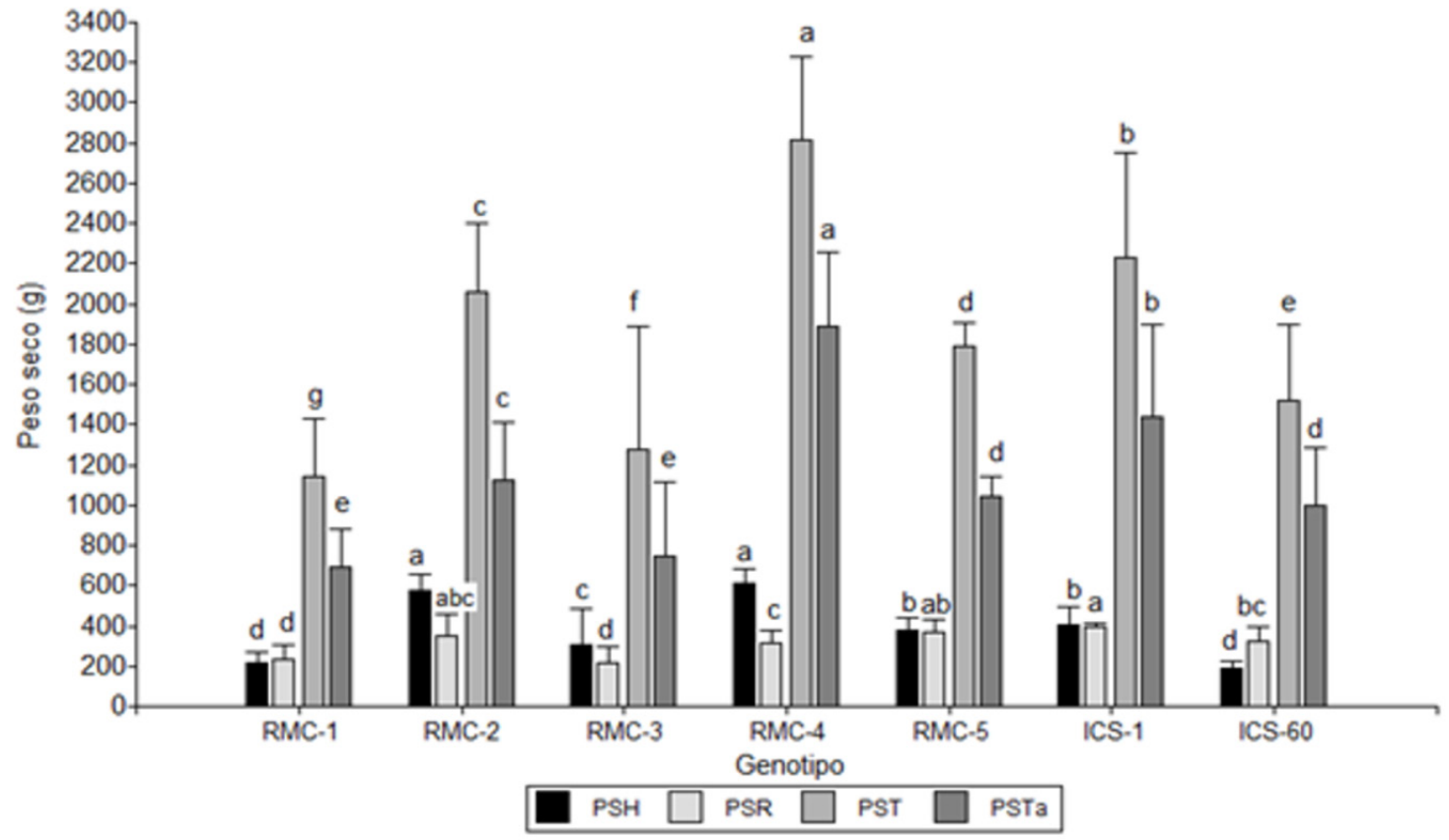




\section{CONCLUSIONES}

Los genotipos promisorios de T. cacao evaluados representan una importante fuente de germoplasma con potencial para su consolidación como clones de rápido desarrollo y producción de biomasa en etapas tempranas de establecimiento, destacándose los genotipos RMC-3, RMC-4 y RMC-5. No obstante, el desempeño agronómico de los genotipos promisorios estuvo condicionado por la distancia de siembra, expresando su mayor potencial a distancia de $4 \times 4 \mathrm{~m}$. Finalmente, se considera necesario el desarrollo de futuros estudios que evalúen la capacidad productiva, el comportamiento fitosanitario de los genotipos promisorios y la producción de biomasa y almacenamiento de $\mathrm{C}$, establecidos en las diferentes distancias de siembra.

\section{AGRADECIMIENTOS}

Al Departamento Administrativo de Ciencia, Tecnología e Innovación (COLCIENCIAS) por la financiación del proyecto de investigación "Desempeño agronómico, evaluación fitosanitaria y caracterización molecular de clones promisorios de Theobroma cacao L. provenientes de materiales vegetales élite presentes en sistemas productivos del Bajo Caguán, municipio de Cartagena del Chairá", código 1131-502-27713 y contrato 719-2011.

\section{REFERENCIAS}

[1] RODRÍGUEZ-MEDINA, CAREN; CAICEDO-ARANA, ALVARO; SOUNIGO, OLIVIER; ARGOUT, XAVIER; ALVARADO-ALVARADO, GABRIEL; YOCKTENG, ROSANA. Cacao breeding in Colombia, past, present and future. Breeding Science, v. 69, n. 3, 2019, p. 373-382.

https://doi.org/10.1270/jsbbs.19011

[2] ZARRILLO, SONIA; GAIKWAD, NILESH; LANAUD, CLAIRE; POWIS, TERRY; VIOT, CHRISTOPER; LESUR, ISABELLE; FOUET, OLIVIER; ARGOUT, XAVIER; GUICHOUX, ERWAN; SALIN, FRANCK; SOLORZANO, REYLORD; BOUCHEZ, OLIVIER; VIGNES, HELENE; SEVERTS, PATRICK; HURTADO, JULIO; YEPEZ, ALEXANDRA; GRIVETTI, LOUIS; BLAKE, MICHAEL; VALDEZ, FRANCISCO. The use and domestication of Theobroma cacao during the mid-Holocene in the upper Amazon. Natura Ecology \& Evolution, v. 2, n. 12, 2018, p. 1879-1888. https://doi.org/10.1038/s41559-018-0697-x

[3] CORNEJO, OMAR E.; YEE, MU-CHING; DOMÍNGUEZ, VÍCTOR; ANDREWS, MARY; SOCKELL, ALEXANDRA; STRANDBERG, ERIKA; LIVINGSTONE III, DONALD; STACK C.; ROMERO, ALBERTO; UMAHARAN, PATHMANATHAN; ROYAERT, STEFAN; TAWARI, NILESH R.; NG, PAULINE; GUTIÉRREZ, OSMAN; PHILLIPS, WILBERT; MOCKAITIS, KEITHANNE; BUSTAMANTE, CARLOS D.; MOTAMAYOR, JUAN C. Population genomic analyses of the chocolate tree, Theobroma cacao L., provide insights into its domestication process. Communications Biology, v. 1, n. 167, 2018, p. 1-12.

http://10.1038/s42003-018-0168-6

[4] RIOS, F.; RUIZ, A.; LECARO, J.; REHPANI, C. Estrategias país para la oferta de cacaos especiales - Políticas e iniciativas privadas exitosas en el Perú, Ecuador, Colombia y República Dominicana. 1 ed. Bogotá D.C. (Colombia): Fundación Swisscontact Colombia, 2017, 140 p.

[5] PERÚ. MINISTERIO DE AGRICULTURA Y RIEGO (MINAGRI). Boletín de divulgación trimestral Enero. Observatorio de commodities: cacao. Lima (Perú): 2019, 10 p.

[6] INICIATIVA LATINOAMERICANA DEL CACAO (ILC). Reflexiones de países miembros de ILAC previo a su participación en la Conferencia Mundial del Cacao. Observatorio del cacao fino y de aroma para América Latina, 3, 2018, p. 2-3.

[7] CÔTE D'IVOIRE. ORGANIZACIÓN INTERNACIONAL DEL CACAO. Boletín Trimestral de Estadísticas del Cacao, tomo XLIII, n1 año cacaotero 2016/17. [on line]. 2017. Disponible: http://siea.minagri.gob.pe/siea/ sites/default/files/1\%20-\%Index-Notes-Market\%20Review\%20(Eng-Fr-Sp)-XLIII-No-1.pdf [citado 4 de Enero de 2020]. 
[8] CÔTE D'IVOIRE. ORGANIZACIÓN INTERNACIONAL DEL CACAO. Boletín Trimestral de Estadísticas del Cacao, tomo XLII, n2 año cacaotero 2015/16. [on line]. 2016. Disponible: http://www.icco.org/home/latest-news.html [citado 4 de Enero de 2020].

[9] CELY-TORRES, LUCY-ANDREA. Oferta productiva del cacao colombiano en el posconflicto. Estrategias para el aprovechamiento de oportunidades comerciales en el marco del acuerdo comercial Colombia-Unión Europea. Equidad \& Desarrollo, v. 28, 2017, p. 167-195.

https://doi.org/10.19052/ed.4211

[10] COLOMBIA. FONDO PARA EL FINANCIAMIENTO DEL SECTOR AGROPECUARIO (FINAGRO). Inteligencia de mercado: cacao. Bogotá (Colombia): 2018, 16 p.

[11] RED DE INFORMACIÓN Y COMUNICACIÓN DEL SECTOR AGROPECUARIO COLOMBIANO (AGRONET). Estadísticas. Agrícola: área, producción, rendimiento y participación [on line]. 2019. Disponible: http://www.agronet.gov.co [citado 5 de marzo de 2020].

[12] COLOMBIA. FEDERACIÓN NACIONAL DE CACAOTEROS (FEDECACAO). Boletín de prensa: pese a las adversidades el 2019 dejó cifras positivas para el sector cacaotero [on line]. 2020. Disponible: http://www. fedecacao.com.co/portal/index.php/es/2015-04-23-20-0033/1103-boletín-de-prensa-pese-a-las-adversidades-el-2019-dejo-cifras-positivas-para-el-sector-cacaotero [citado 24 de febrero de 2020].

[13] HOLDRIDGE, LESLIE-RENSSELAER. Life zone ecology. 1 ed. San José (Costa Rica):Tropical Science Center, 1967, 206 p.

[14] DI RIENZO, J.A.; CASANOVES, F.; BALZARINI, M.G.; GONZÁLEZ, L.; TABLADA, M.; ROBLEDO, C.W. InfoStat versión 2018 [online]. 2018. Disponible: http://www.infostat.com.ar [citado 4 de Febrero de 2020].

[15] DI RIENZO, JULIO A.; MACCHIAVELLI, RAÚ L.; CASANOVES, FERNANDO. Modelos lineales generalizados mixtos: aplicaciones en InfoStat. 1 ed. Especial. Córdoba (Argentina): Grupo Infostat, 2017, 104 p.

[16] PINHEIRO, JOSÉ; BATES, D.; DEBROY, S.; SARKAR, D. R. Core Team (2017) nlme: linear and nonlinear mixed effects models. R Package Version 3.1-131. [on line]. 2017. Disponible: https://CRAN. R-project. org/package=nlme [citado 4 de Febrero de 2020].

[17] R. CORE TEAM. R. A Language and Environment for Statistical Computing [on line]. 2018. Disponible: https://cran.r-project.org/ [citado 4 de Enero de 2020].

[18] SARMIENTO, SINDY-CAROLINA; GAMBOA-TABARES, JEAN-ALEXANDER; VELASQUEZ -RESTREPO, JAIME-ENRIQUE. Desempeño agronómico de tres clones de cacao en fase de vivero en la Amazonia Colombiana. Ingeniería \& Amazonia, v. 4, n. 1, 2011, p. 39-47.

[19] CORTÉS-PATIÑO, SANDRA-LUCIA; VESGA-AYALA, NELCY P.; SIGARROA-RIECHE, ALINA K.; MORENO-ROZO, LAURA; CÁRDENAS-CARO, DIANA. Sustratos inoculados con microorganismos para el desarrollo de plantas de cacao (Theobroma cacao L.) en etapa de vivero. Bioagro, v. 27, n. 3, 2015, p. 151-158.

[20] ANITA-SARY, INDAH; WAHYU-SUSILO, DAN-AGUNG. Keberhasilan sambungan pada beberapa jenis batang atas dan famili batang bawah kakao (Theobroma cacao L.) Pelita Perkebunan, v. 28, n. 2, 2012, p. 72-81.

[21] PALENCIA, GILDARDO; MEJÍA, LUIS A. Injertación temprana en la producción masiva de clones de cacao. 1 ed. Bucaramanga (Colombia): Corporación Colombiana de Investigación Agropecuaria, CORPOICA, 2004, 38 p.

[22] PALENCIA-CALDERÓN, GILDARDO-EFRAIN; GÓMEZ-SANTOS, RAUL; GUIZA, ORLANDO. Nuevas tecnologías paras instalar viveros y producir clones de cacao (Theobroma cacao L.). 1 ed. Bogotá D.C. (Colombia): Produmedios, 2009, $31 \mathrm{p}$.

[23] POVEDA, VERÓNICA; OROZCO, LUIS; MEDINA, CRISTÓBAL; CERDA,ROLANDO; LÓPEZ, ARLENE. AImacenamiento de carbono en sistemas agroforestales de cacao en Waslala, Nicaragua. Agroforestería de las Américas, v. 49, 2013, p. 42-50.

[24] ZABALA, WILFRIDO; MERINO, ERICA; PEÁLEZ, PEDRO. Influencia de tres sistemas agroforestales del cultivo de cacao en la captura y almacenamiento de carbono. Scientia Agropecuaria, v. 9, n. 4, 2018, p. 493-501. http://dx.doi.org/10.17268/sci.agropecu.2018.04.04

[25] TAGNANG-NADĖGE, MADOUNTSAP; LOUIS, ZAPFACK; CHIMI-DJOMO, CÉDRIC; LOUIS-PAUL, KABELONG-BANOHO; PREASIOUS-FUNWI, FORBI; TSOPMEJIO-TEMFACK, INGRID; VICE-CLOTEX, TAJEUKEM; NTONMEN-YONKEU, AMANDINE-FLORE; TABUE-MBOBA, ROGER-BRUNO; JULLIETE, NASANG. Carbon storage potential of cacao agroforestry systems of different age and management intensity. Climate and Development, v. 11, n. 7, 2018, p. 543-554.

https://doi.org/10.1080/17565529.2018.1456895 